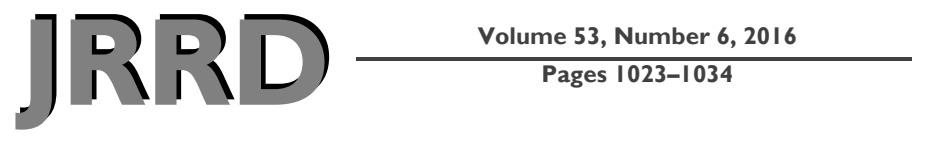

\title{
Efficacy of extremely low-frequency magnetic field in fibromyalgia pain: A pilot study
}

\author{
Teresa Paolucci, MD, PhD; ${ }^{*}$ Giulia Piccinini, MD; ${ }^{1}$ Marco Iosa, PhD; ${ }^{2}$ Cristina Piermattei, MD; ${ }^{1}$ Simona de \\ Angelis, MD; ${ }^{1}$ Maria Rosaria Grasso, MD; ${ }^{1}$ Federico Zangrando, FT; ${ }^{1}$ Vincenzo Maria Saraceni, MD $^{\mathbf{1}}$ \\ ${ }^{1}$ Complex Operative Unit in Physical Medicine and Rehabilitation, Azienda Policlinico Umberto I, Sapienza University \\ of Rome, Rome, Italy; ${ }^{2}$ Clinical Laboratory of Experimental Neurorehabilitation, Santa Lucia Foundation, Rome, Italy
}

\begin{abstract}
The purpose of this pilot study was to determine the efficacy of an extremely low-frequency magnetic field (ELF-MF) in decreasing chronic pain in fibromyalgia (FM) patients. Thirty-seven females were recruited and randomized into two groups: one group was first exposed to systemic ELFMF therapy (100 microtesla, 1 to $80 \mathrm{~Hz}$ ) and then to sham therapy, and the other group received the opposite sequence of intervention. Pain, FM-related symptoms, and the ability to perform daily tasks were measured using the Visual Analog Scale, Fibromyalgia Impact Questionnaire (FIQ), Fibromyalgia Assessment Scale (FAS), and Health Assessment Questionnaire (HAQ) at baseline, end of first treatment cycle, beginning of second treatment cycle (after 1 mo washout), end of second treatment cycle, and end of 1 mo follow-up. ELF-MF treatment significantly reduced pain, which increased on cessation of therapy but remained significantly lower than baseline levels. Short-term benefits were also observed in FIQ, FAS, and HAQ scores, with less significant effects seen in the medium term. ELF-MF therapy can be recommended as part of a multimodal approach for mitigating pain in FM subjects and improving the efficacy of drug therapy or physiotherapy.
\end{abstract}

Clinical Trial Registration: ClinicalTrials.gov; "Very low frequency magnetic fields in the treatment of fibromyalgia": NCT02231541; $\quad$ https://clinicaltrials.gov/ct2/show/ NCT02231541?term $=$ NCT02231541\&rank $=1$

Key words: chronic pain, electromagnetic fields, ELF, extremely low-frequency, fibromyalgia, magnetic fields, magnetotherapy, pain, physical therapy, rehabilitation.

\section{INTRODUCTION}

Fibromyalgia (FM) is a chronic condition that is characterized by widespread body pain (present for more than 3 mo, above and below the waist, on the left or right side of the body) and pain on digital palpation of at least 11 of 18 predefined tender points. The prevalence of FM in the general population is estimated to be 2 to 7 percent. The chronic pain in FM is often associated with comorbidities, such as fatigue, depression, sleeping disorders, morning stiffness, irritable bowel syndrome, diffuse abdominal pain, anxiety, and headache [1-2].

Although the pathogenesis of FM is not completely understood, it has been suggested that peripheral or central

\footnotetext{
Abbreviations: $\mathrm{ELF}=$ extremely low-frequency, FAS = Fibromyalgia Assessment Status, FIQ = Fibromyalgia Impact Questionnaire, $\mathrm{FM}=$ fibromyalgia, $\mathrm{HAQ}=$ Health Assessment Questionnaire, MF $=$ magnetic field, $\mathrm{PEMF}=$ pulsed electromagnetic field, $\mathrm{T} 0=$ baseline, $\mathrm{T} 1=$ end of first treatment cycle, $\mathrm{T} 2$ = beginning of second treatment cycle (after $1 \mathrm{mo}$ washout), $\mathrm{T} 3=$ end of second treatment cycle, $\mathrm{T} 4=$ after $1 \mathrm{mo}$ follow-up, TMS = transcranial magnetic stimulation, VAS = Visual Analog Scale.

*Address all correspondence to Teresa Paolucci, MD, PhD; Complex Operative Unit in Physical Medicine and Rehabilitation, Azienda Policlinico Umberto I, Sapienza University of Rome, Piazzale Aldo Moro 5, 00185, Rome, Italy; 0039-347-9338625; fax: 0039-0649914552.
}

Email: teresapaolucci@hotmail.com http://dx.doi.org/10.1682/JRRD.2015.04.0061 
hyperexcitability at the level of the spinal cord or brain stem, changes in pain perception, and somatization mitigate the pain. Several studies have implicated central pain sensitization of the brain pain matrix in the pathogenesis of chronic pain [3-7].

The etiopathology of FM is considered to be multifactorial and develops through the interaction of neurohormonal, genetic, and psychological factors. Conversely, no FM-specific personality has been defined [8], and personality has been proposed to be another important filter that modulates one's response to psychological stressors. Certain personalities facilitate the translation of such stressors into physiological responses, driving fibromyalgic mechanisms [9].

Physical exercise and multimodal cognitive behavioral therapy are the most widely accepted and beneficial forms of nonpharmacological treatment for FM [10-14]. Yet, there is equivocal evidence regarding the efficacy of physical therapy in FM. Chiropractic, laser therapy, magnetic field (MF) therapy, massage, and transcranial current stimulation are not recommended, based on a recent review by Winkelmann et al. [15]. Alternatively, other studies have demonstrated relief from FM symptoms through laser therapy [16]. MF therapy has been applied to treat osteoarthritis and inflammatory diseases of the musculoskeletal system, alleviate pain, accelerate the healing of ulcers, and reduce spasticity [17]. There is evidence of the effects of MFs on brain signals and certain psychological disorders, such as headache, migraine, and depression. Based on these findings, specific protocols can be designed using a combination of exposures to various MFs that generate the brain signals necessary to clinically evaluate the effects of MFs [18-19].

Extremely low-frequency (ELF)-MFs in the picotesla and millitesla ranges are administered to improve neurotransmission and correct local immune pathology, respectively [20]; they are effective in decreasing chronic pain in osteoarthritis and reducing fatigue in multiple sclerosis.

ELF-MFs alter animal behavior and modulate biological variables, including gene expression, cell survival, cellular differentiation, and cerebral blood flow in aged transgenic mice [21-22]. Alterations in inflammatory responses have also been observed, but how these activities affect human health remains unknown [23].

Other studies have indicated a beneficial effect of ELF-MFs in a model of global cerebral ischemia, inhibiting vessel growth in a specific range of amplitudes and thus demonstrating antiangiogenic activity [24-25].
Although the precise mechanism of ELF-MFs remains undetermined, they have unexpected short-term analgesic effects in neuropathic pain [26-27]. No study has examined the efficacy of ELF-MFs in FM, excluding reports on transcranial pulsed MFs [28-31].

Shupak and colleagues studied specific pulsed electromagnetic fields (PEMFs) in FM that extended from the outer periphery of the cingulate cortex to the brain midline (30 min duration, 200 to $400 \mu \mathrm{T}, 1 \mathrm{kHz}$ ); PEMFs effected a modest reduction in pain in patients with rheumatoid arthritis but not for those with FM versus the sham group [28]. In contrast, Maestú and colleagues studied the effect of very low-intensity pulsed transcranial magnetic stimulation (TMS) on FM (once per week, 8 sessions, 20 min duration, $43 \mathrm{nT}$ for each coil, $8 \mathrm{~Hz}$ ) and noted that it had analgesic and antinociceptive effects, similar to the opioid analgesic effects in PEMFexposed patients [29]. Nevertheless, there is no definitive treatment modality that is effective in FM patients, and the results are often mixed.

Based on these studies, we wanted to expand the use of nonpulsed ELF-MFs through total body magnetic exposure as opposed to TMS. The aim of this pilot study was to determine the efficacy of mild nonpulsed ELFMFs in mitigating chronic pain in FM patients.

\section{MATERIALS AND METHODS}

\section{Study Design}

This crossover, randomized, double-blind pilot study measured the effects of nonpulsed ELF-MFs versus sham therapy on chronic pain in subjects with FM (Figure 1). All patients underwent a period of ELF-MF therapy and a period of sham therapy, half of them in that sequence and the other half receiving sham treatment first.

\section{Subjects}

From September 2014 to December 2014, 37 female subjects were recruited from the Physical Medicine and Rehabilitation outpatient clinic, Policlinico Umberto I Hospital, Sapienza University of Rome, Rome, Italy. The mean age (in years) was $49.50 \pm 9.38$ and $51.12 \pm 12.47$ for the ELF-MF and sham groups, respectively; the mean body mass index values (kilogram per meter square) were $24.89 \pm 5.26$ and $25.85 \pm 6.43$, respectively. All subjects' FM was defined clinically per the 1990 and 2010 American College of Rheumatology criteria. We 


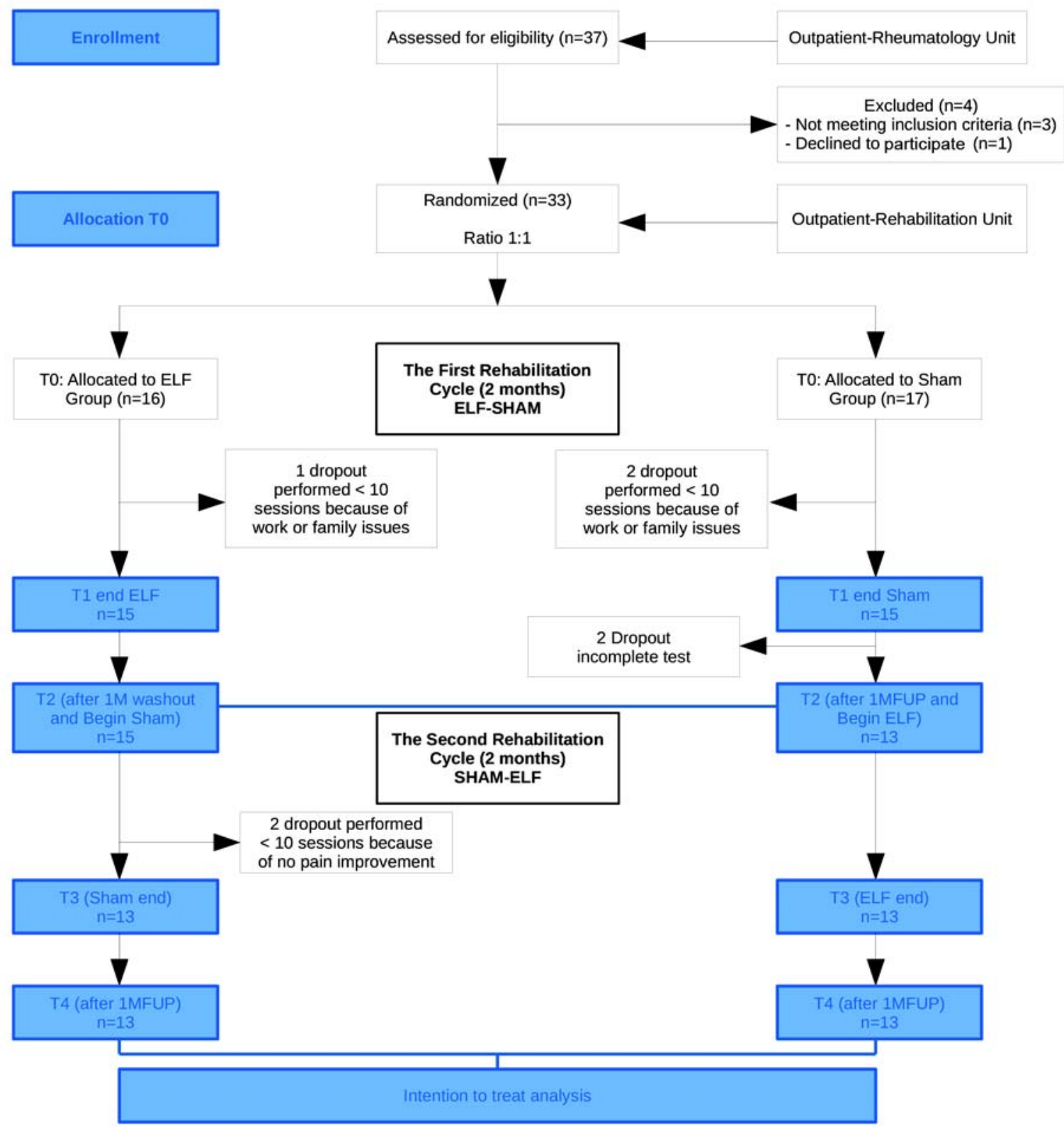

Figure 1.

Flowchart of the study. ELF = extremely low-frequency, 1MFUP = 1 mo follow-up, T0 = baseline, T1 = end of first treatment cycle, T2 = beginning of second treatment cycle (after 1 mo washout), T3 = end of second treatment cycle, T4= after 1 mo follow-up.

included all subjects with FM who experienced widespread pain for more than 3 mo and pain with $4 \mathrm{~kg} / \mathrm{cm}^{2}$ pressure at 11 or more of the 18 tender points (in every case, the diagnosis of FM had been established by the patient's rheumatologist), were aged 18 to $60 \mathrm{yr}$, and had a Visual Analog Scale (VAS) score $>3$ for pain.
The exclusion criteria were the presence of concomitant autoimmune or hematologic diseases, psychiatric disorders (such as mild depression and anxiety with pharmacological and psychological treatment), other causes of chronic pain, and other diseases such as epilepsy and tumors. Pregnant women, those with pacemakers, and 
subjects who were concurrently participating in another type of physical therapy were excluded. Also, subjects with overlapping painful conditions, such as chronic fatigue and irritable bowel or inflammatory bowel syndrome, were excluded.

Those with comorbidities, such as myocardial infarction, lower-limb arterial disease, major neurological problems, diabetes, gastrointestinal disease, chronic respiratory disease, kidney disease, and poor vision, were not included. The pharmacological therapeutic regimen must have been stable for at least 3 mo before the patient began treatment: acetaminophen up to $3 \mathrm{~g} / \mathrm{d}$, tramadol up to $200 \mathrm{mg} / \mathrm{d}$, and pregabalin up to $150 \mathrm{mg} / \mathrm{d}$.

All patients were instructed not to take any new medications during the study protocol and to avoid other rehabilitation approaches. During the rehabilitation sessions, no patient reported an increase in pain that led to treatment discontinuation or greater use of current drug therapy. Overall, 3 of the initial 37 recruited subjects were excluded and 1 declined to participate. We also excluded patients who attended fewer than 10 sessions $(n=7)$.

Patients were randomized into two groups. One group consisted of 16 patients with FM who were exposed to ELF-MFs first and then received sham exposures. The other group consisted of 17 patients with FM who received sham exposures first and were then exposed to ELF-MFs. There were no significant differences in baseline characteristics between groups. For ethical consideration, each group underwent ELF-MF treatment.

\section{Magnetic Field Treatment and Setting}

The LIMFA system (Eywa srl; Rimini, Italy) (ISO9001 certification number 390263) was used to create multifrequency magnetoelectric fields with an intensity of $100 \mu \mathrm{T}$ and a low-frequency field.

Subjects participated in 12 treatment sessions, 3 times per week for 4 weeks, in a double-blind controlled trial, with each session lasting for $30 \mathrm{~min}$. Patients were asked to rest on a bed on a multi-low-frequency MF mattress and exposed to genuine or sham therapy. Genuine therapy comprised systemic ELF-MF with an intensity of $100 \mu \mathrm{T}$ and a multifrequency of 1 to $80 \mathrm{~Hz}$ (Figure 2).

The device, a magnetic mattress, works during sham or genuine exposure depending on the type of modality that is specified. The observer and patient were blinded to the modality that was activated. Active and placebo

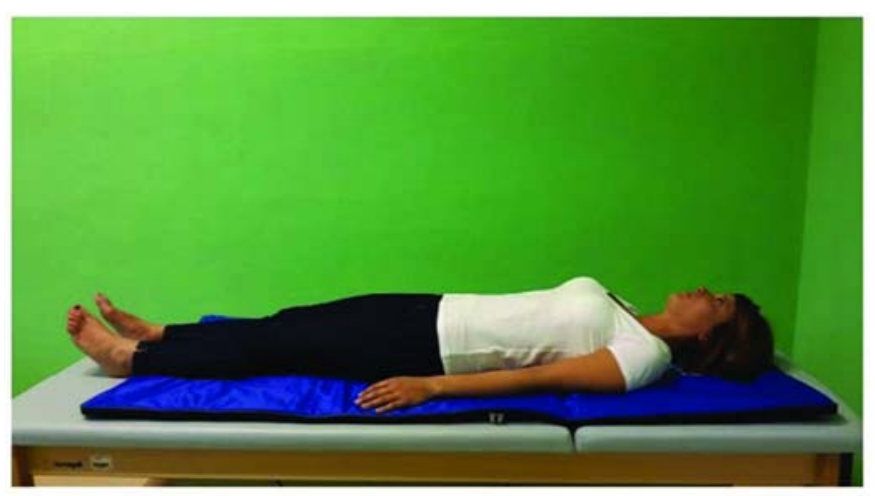

Figure 2.

Treatment with extremely low-frequency magnetic field.

codes were randomly assigned to the groups and revealed on completion of the study by all participants. The sham modality was obtained by switching to a different code on the device, resulting in no magnetic fields being generated, as if the machine were turned off.

\section{Outcome Measures}

The primary outcome was the change in chronic pain. Subjects were evaluated at baseline (T0), at the end of the first treatment cycle (T1), at the beginning of the second treatment cycle (after a 1 mo washout) (T2), at the end of the second treatment cycle (T3), and after 1 mo follow-up (T4). T2 was considered the crossover point for the two groups (Figure 1).

Patients were evaluated with specific FM scales. The Fibromyalgia Impact Questionnaire (FIQ) consists of three sections: Function, Impact, and Symptoms, which when combined produce an overall score. The first section contains 10 subitems (FIQ-Physical Impairment) and focuses on the patient's ability to perform daily tasks that involve the large muscles (e.g., cooking, cleaning, walking, shopping, homemaking, socializing, and mobility). The next two sections (FIQ-Feel Good and FIQ-Work Missed) ask patients to circle the number of days in the past week on which they felt good and the number of days that they missed work. The last seven items probe the ability to do one's job, pain, fatigue, morning tiredness, stiffness, anxiety, and depression. The total FIQ score is calculated by adding the following 10 items: the physical functioning score, the number of days of feeling good, the number of work days missed, the ability to do one's job, pain, fatigue, morning tiredness, stiffness, anxiety, and 
depression. The FIQ score ranges from 0 to 100 , with 100 indicating the maximum impact of FM [32]. The FIQ has been translated into many languages, including Italian [33].

The Fibromyalgia Assessment Status (FAS) is a simple and rapidly implemented index consisting of a "pain map" called the Self-Assessment Pain Scale (in which the patient is asked to indicate how much pain he or she suffered in the previous week in 16 areas of the body, with a grading scale that ranges from 0 to 3 ) and two scales (with ratings between 0 and 10) that evaluate fatigue and quality of sleep, for a total score of 0 to 10 . The FAS allows physicians to obtain reliable information concerning the course of the disease and is sensitive enough to alert them in the case of deterioration [34].

The Health Assessment Questionnaire (HAQ) is a self-administered, 20-item questionnaire that assesses difficulties in performing eight daily activities (dressing and grooming, getting up, eating, walking, hygiene, reaching, ability to grip, and outside activities). For each item, patients are asked to rate the level of difficulty that they have experienced over the previous week in performing these activities on a 4-point scale, from 0 (no difficulty) to 3 (unable to perform). The final HAQ score is the average score of the eight categories and thus also ranges from 0 to 3 , with higher scores reflecting greater disability [35].

The VAS is a simple, robust, sensitive, and reproducible instrument that enables patients to express their pain intensity as a numerical value. Patients were asked to mark the point that corresponded to their perceived pain intensity on a $10 \mathrm{~cm}$ line, with 0 indicating the absence of pain and 10 reflecting the most severe pain [36].

\section{Sample Size and Statistical Analysis}

Because this trial was a pilot study, no sample size was determined. The data are expressed as mean and standard deviation. Because the clinical scores are ordinal numbers, nonparametric statistics were chosen for the between- and within-group analyses. Percentage improvement with respect to beginning of the treatment for the periods in which patients received ELF-MF and sham treatment was compared and analyzed using MannWhitney $U$-test. Within-group comparisons were performed using Friedman analysis, followed by Wilcoxon signed-rank test, for each group with regard to changes in scores from baseline levels (T0). The alpha level was set to 0.05 for all analyses, with the exception of post hoc following Friedman analysis, for which Bonferroni cor- rection was applied. An intention-to-treat analysis was performed.

\section{RESULTS}

As shown in Figure 1, 33 of 37 subjects who were screened for eligibility were enrolled into the study; 16 patients underwent ELF-MF and then sham treatment, and 17 received the therapies in reverse order. Baseline scores (T0) did not differ significantly for any scale. Patient characteristics at baseline are listed in Table $\mathbf{1 .}$ Thirty participants completed at least one treatment cycle, and their data were analyzed. Throughout the study, 7 patients dropped out; thus, 26 patients ultimately completed the entire protocol.

The primary outcome measure was reduction in pain, assessed by VAS (Figure 3). ELF-MF treatment significantly reduced pain $(p=0.001)$, which rose after the end of treatment but remained significantly lower than baseline levels $(p=0.001)$. Short-term benefits were also observed in terms of the secondary outcome measures, but the medium-term effects were less significant.

Figure 4 shows the changes in FAS scale scores: significant improvements in FAS scores were noted at the end of treatment ( $\mathrm{T} 1$ for the ELF-sham group, T3 for sham-ELF group) and lasted 1 mo (T2 and T4, respectively), becoming nonsignificant at $\mathrm{T} 3$ and $\mathrm{T} 4$ for the ELF-sham group.

The HAQ scores are reported in Figure 5. Only ELFsham patients had significantly different scores than their counterparts who received sham therapy at the end of treatment $(p<0.001$ at $\mathrm{T} 1)$; this change was poorly maintained after $1 \mathrm{mo}(p=0.03$ at T2, not significantly different from T0 after Bonferroni correction). Analogously, the sham-ELF group showed a significant within-group effect of ELF-MF only at 1 mo after the end of treatment (T4).

The within-group declines in FIQ score that were induced by ELF-MF were significant in both groups (Figure 6). FIQ scores recovered only after T2 in ELFsham patients, despite remaining significantly lower than baseline scores at T3, but this significance was lost at T4. In comparing FIQ subscores for the ELF-sham versus sham-ELF groups at T1 and T2, we found that the significant changes in overall scores were attributed to improvements in FIQ-Physical Impairment $(p=0.03$ at $\mathrm{T} 1, p<0.001$ at T2), FIQ-Feel Good $(p=0.02$ and $p<$ 0.001 , respectively), FIQ-Work Missed ( $p=0.003$ for 
JRRD, Volume 53, Number 6, 2016

Table 1.

Baseline demographics and clinical characteristics of the study population with fibromyalgia (FM) and relevant means and standard deviations (SDs) for the extremely low-frequency (magnetic field) (ELF)-sham group, sham-ELF group, and entire sample (pharmacological regimen [PR]: acetaminophen-tramadol $=\mathrm{A}$, pregabalin $=\mathrm{B}$, nothing $=0)$.

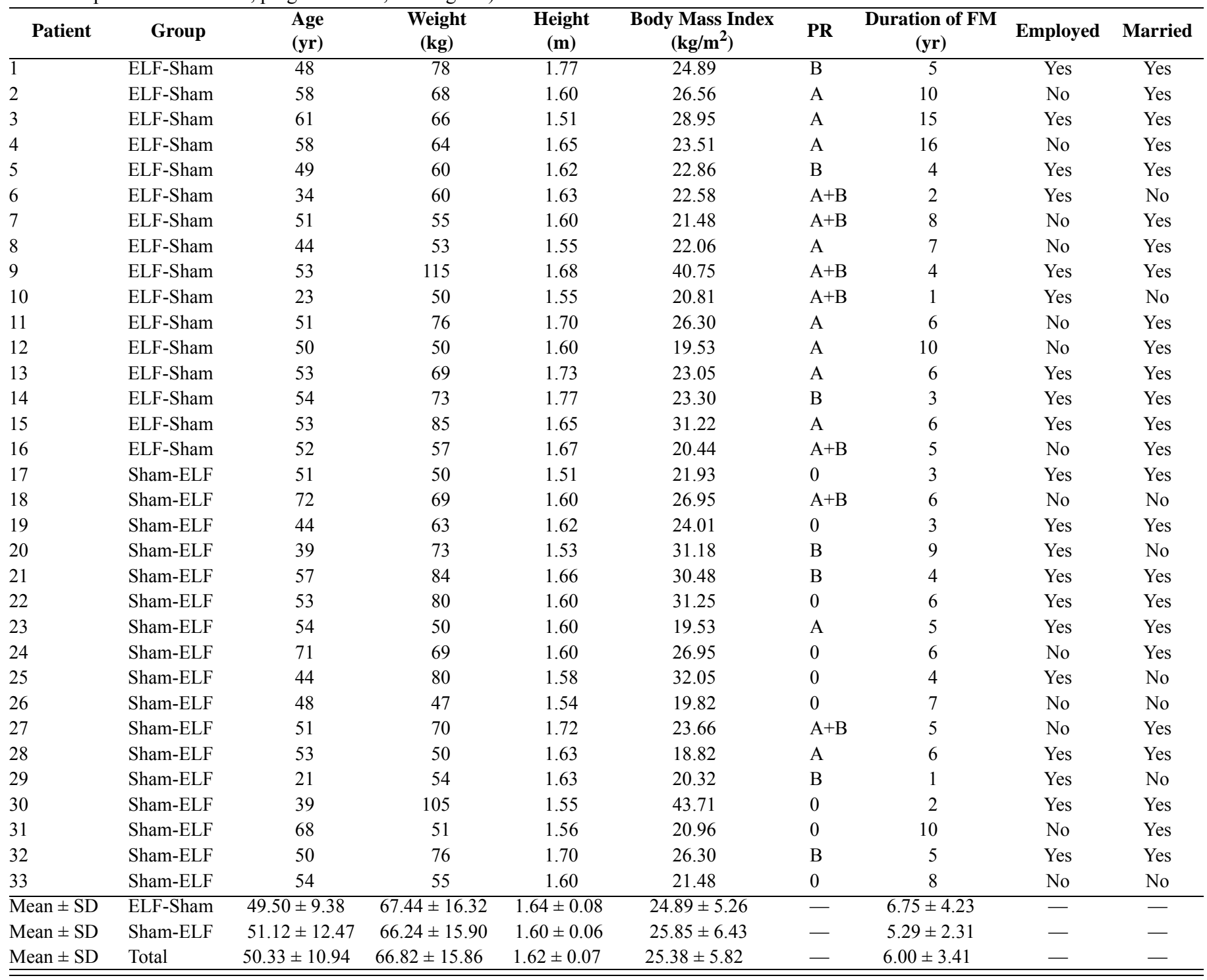

both), FIQ-Pain ( $p=0.005$ and $p<0.001$, respectively), FIQ-Fatigue ( $p=0.003$ and $p<0.001$, respectively), FIQ-Morning Tiredness $(p=0.001$ and $p<0.001$, respectively), FIQ-Stiffness ( $p=0.004$ and $p<0.001$, respectively), and FIQ-Depression ( $p=0.003$ and $p<0.001$, respectively). In contrast, differences in FIQ-Anxiety scores were not significant at T1 $(p=0.07)$ but became so at T2 $(p=0.001)$. A summary of the scale scores is listed in terms of mean and standard deviation and $p$ values by Mann-Whitney $U$-test (in bold if statistically significant) (Table 2).
Table 3 summarizes the data as percentage improvement with respect to beginning of the treatment for patients with ELF-MF and sham treatment. VAS scores generally declined by 50 percent versus 40 percent for FAS and FIQ scores between pre- and post-ELF-MF treatment (i.e., T1 vs T0 in the ELF-sham group and T3 vs T2 in the sham-ELF group). These values were higher than what was observed with the sham treatment, which was approximately -7 percent, -6 percent, and -18 percent, respectively (average comparisons between $\mathrm{T} 3$ and $\mathrm{T} 2$ in 


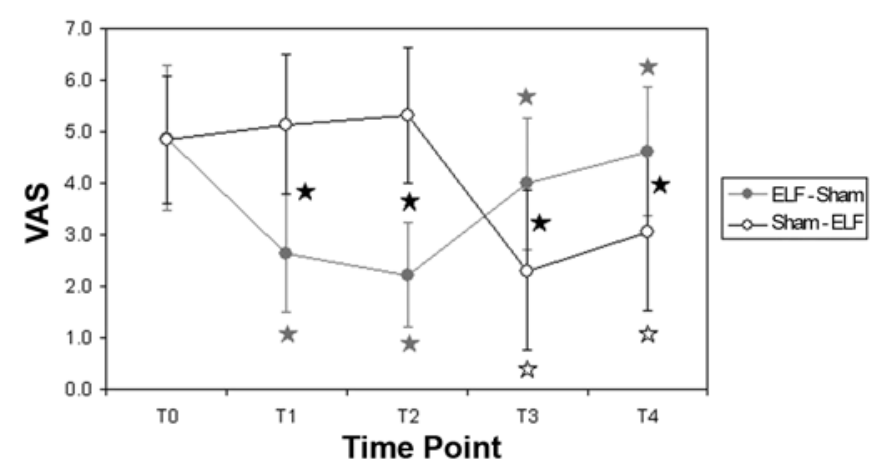

Figure 3.

Mean and standard deviation of Visual Analog Scale (VAS) for pain for patients who performed extremely low-frequency (magnetic field) (ELF) and then sham treatment (gray) or the opposite (empty circles). Statistically significant differences are shown with stars: filled black stars for between-group comparisons, gray stars for within-group comparisons with respect to TO values for ELF-sham group, black empty stars for withingroup comparisons with respect to T0 values for sham-ELF group. $\mathrm{T0}=$ baseline, $\mathrm{T} 1=$ end of first treatment cycle, $\mathrm{T} 2=$ beginning of second treatment cycle (after 1 mo washout), T3 = end of second treatment cycle, T4 = after 1 mo follow-up.

the ELF-sham group and T1 and T0 in the sham-ELF group). No side effects were recorded during the study.

\section{DISCUSSION}

We noted good efficacy of ELF-MFs compared with placebo. With regard to our primary outcome, as assessed by the VAS, ELF-MF treatment significantly reduced pain, which increased at the end of treatment but remained significantly lower than baseline levels $(p=$ $0.001)$. The VAS results are consistent with reported minimal clinically important difference values of a 23 percent to 35 percent improvement in pain versus baseline values [37-38]. Short-term benefits were also observed in secondary outcome measures, but the medium-term effects were less significant.

Nevertheless, our results are encouraging and should prompt a continuing investigation of ELF-MF exposure for short-term pain relief in FM patients and the application of this stimulation over the long term. Future studies should compare our ELF-MF protocol (which had benefits without any side effects) with other more intensive programs, for example, daily treatment or doubling the

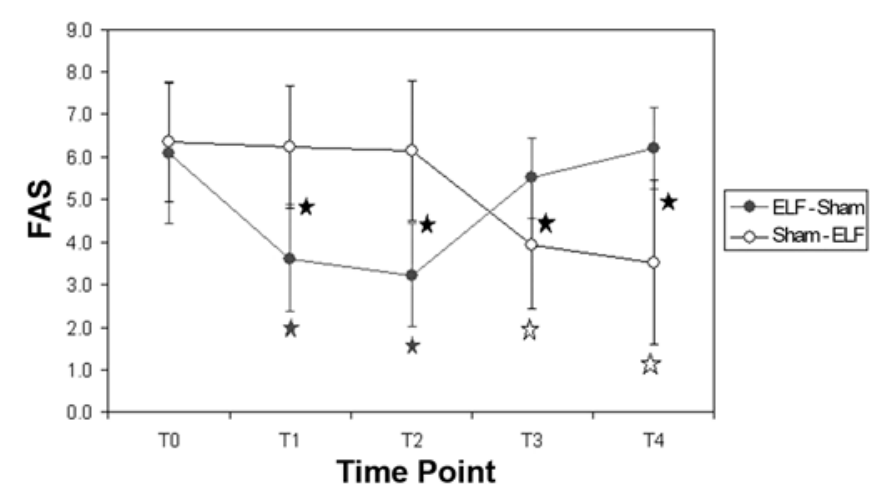

Figure 4.

Mean and standard deviation of Fibromyalgia Assessment Scale (FAS) scores for patients who performed extremely lowfrequency (magnetic field) (ELF) and then sham treatment (gray) or the opposite (empty circles). Statistically significant differences are shown with stars: filled black stars for between-group comparisons, gray stars for within-group comparisons with respect to TO values for ELF-sham group, black empty stars for within-group comparisons with respect to T0 values for shamELF group. T0 = baseline, $\mathrm{T} 1$ = end of first treatment cycle, $\mathrm{T} 2=$ beginning of second treatment cycle (after 1 mo washout), T3 = end of second treatment cycle, T4 = after 1 mo follow-up.

number of sessions in a single day. Existing TMS protocols for the treatment of pain in FM differ in frequency (nearly always pulsed), intensity, duration, and setting [28-31]; no standardized protocol for ELF-MF treatment in FM has been developed, and there are no studies in this area with respect to total body stimulation with the magnetic mattress. Consistent with similar studies, our results demonstrate an analgesic and antinociceptive effect, similar to the opioid analgesic effect in PEMFexposed patients [39-40].

A limitation of our study was the lack of a biochemical assessment of the effects of ELF-MF on pain relief in our FM patients. We hypothesize that the opioid analgesic effect is related to the central sensitization that characterizes FM. One of the hallmarks of FM is the implementation of sensory input that is mediated by central nervous system events, similar to neuropathic pain conditions (i.e., central sensitization [increases in Substance P, a neuronal excitatory substance that mediates the conduction of pain in the central nervous system]). FM has also been proposed to involve a reduction in serotonin (a neurotransmitter of the inhibitory descending system) and abnormal levels of norepinephrine, which modulates endogenous pain inhibitory 
JRRD, Volume 53, Number 6, 2016

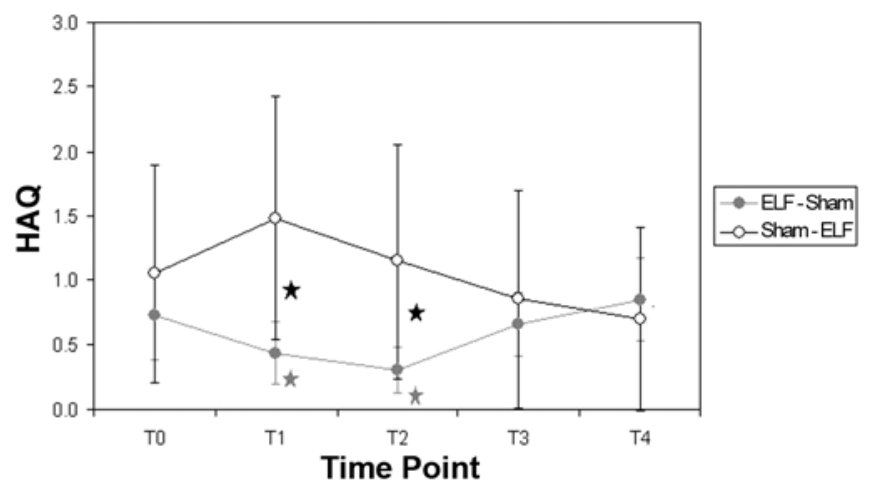

Figure 5.

Mean and standard deviation of Health Assessment Questionnaire (HAQ) scores for patients who performed extremely lowfrequency (magnetic field) (ELF) and then sham treatment (gray) or the opposite (empty circles). Statistically significant differences are shown with stars: filled black stars for between-group comparisons, gray stars for within-group comparisons with respect to T0 values for ELF-sham group, black empty stars for within-group comparisons with respect to TO values for ShamELF group. T0 = baseline, $\mathrm{T} 1=$ end of first treatment cycle, $\mathrm{T} 2=$ beginning of second treatment cycle (after 1 mo washout), T3 = end of second treatment cycle, T4 = after 1 mo follow-up.

pathways and affects cortisol suppression [41]. The central augmentation of sensory input is associated with enhanced sensitivity to pain. Also, the chronic pain and allodynia in FM as well as in mood disorders are associated with significantly lower levels of ATP in platelets, which has been implicated in their pathogenesis [42-43]. Likely, ELF-MF reduces pain by relieving peripheral input in FM patients and has short latency effects $(1 \mathrm{mo})$, even after the end of treatment and despite the modulation of biochemical mediators of pain, maintaining a short biochemical memory [44].

The algogenic effect of electromagnetic fields has also been observed with pulsed MFs in osteoarthritic disease [45-46]. Musaev and colleagues reported that lowfrequency pulsed MFs have analgesic, vasoactive, neuronstimulating, and trophic effects in patients with diabetic polyneuropathy, which has a similar sensory profile as FM [26].

The mechanism of the effect of MF therapy on pain remains unknown, but certain studies have shown that shortterm exposure to electromagnetic fields influences several inflammatory cellular and neurological processes, such as patterns of cortical activation and inhibition and the activity of various neurotransmitters, as in multiple sclerosis [40].

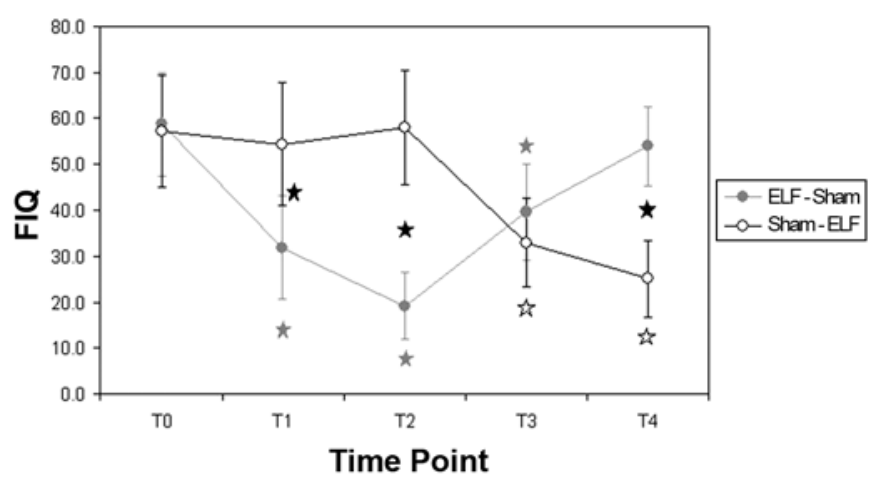

Figure 6.

Mean and standard deviation of Fibromyalgia Impact Questionnaire (FIQ) scores for patients who performed extremely lowfrequency (magnetic field) (ELF) and then sham treatment (gray) or the opposite (empty circles). Statistically significant differences are shown with stars: filled black stars for between-group comparisons, gray stars for within-group comparisons with respect to TO values for ELF-sham group, black empty stars for within-group comparisons with respect to T0 values for shamELF group. T0 $=$ baseline, $\mathrm{T} 1=$ end of first treatment cycle, $\mathrm{T} 2=$ beginning of second treatment cycle (after 1 mo washout), T3 = end of second treatment cycle, T4 = after 1 mo follow-up.

Our results do not demonstrate a stabilization of the effect at the end of treatment. FIQ scores worsened after T2 in ELF-sham patients, despite remaining significantly lower than baseline levels at T3, but this significance was lost at T4. These results might be due to the cyclical nature of pain that characterizes patients with FM and to our use of a nonintensive ELF-MF protocol in terms of duration of treatment, weekly frequency, and number of sessions. Because this trial was a pilot study, with no specific reference protocols, we decided to adopt a nonintensive treatment protocol to better respect the parameters of patient safety. However, the source of sensory input in FM patients remains unknown; thus, the duration of the efficacy of ELF-MF remains undetermined. Similarly, the magnetite hypothesis, based on the induction of electric currents, appears to be an unlikely mechanism, given that the induced fields are orders of magnitude lower than the endogenous electric fields in tissues; a connection between magnetite and the nervous system has not been demonstrated [39-40,47].

Sleep quality improved, as reflected in the FIQ subscales, with ELF-MF. As an MF therapy, ELF-MF acts 
Table 2.

Group comparisons. Scores are expressed as mean \pm standard deviation; $p$-values in the rows refer to Mann-Whitney $U$-test, and those in the last column were calculated by Friedman analysis for each group over time (in bold if statistically significant).

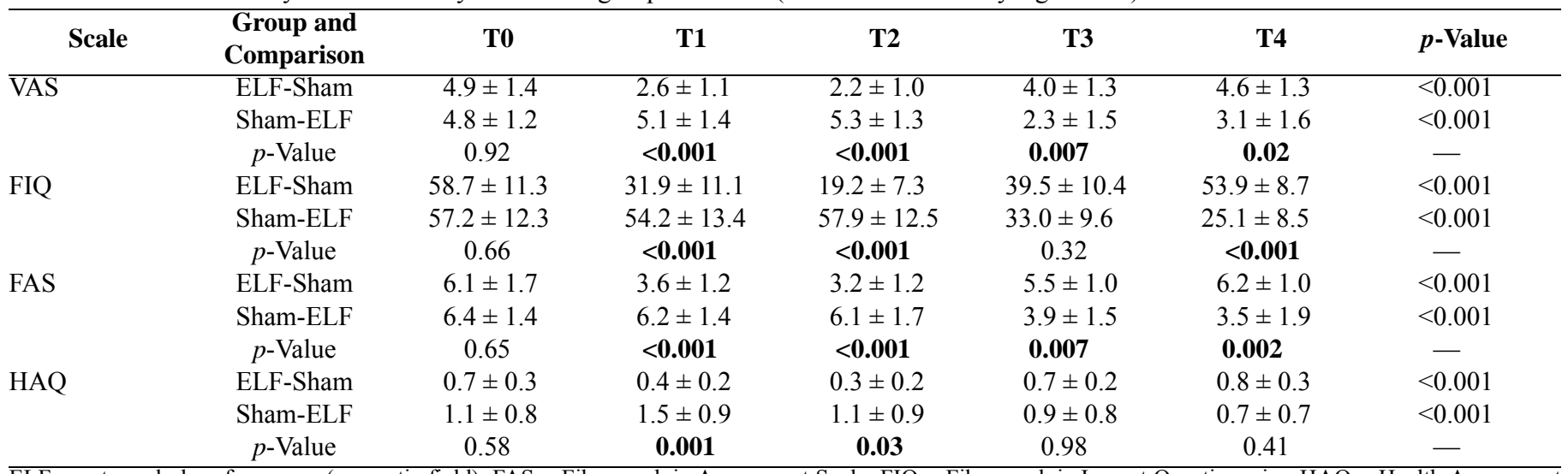

$\overline{\mathrm{ELF}}=$ extremely low-frequency (magnetic field), FAS = Fibromyalgia Assessment Scale, FIQ = Fibromyalgia Impact Questionnaire, HAQ = Health Assessment Questionnaire, T0 = baseline, $\mathrm{T} 1=$ end of first treatment cycle, $\mathrm{T} 2=$ beginning of second treatment cycle (after 1 mo washout), $\mathrm{T} 3=$ end of second treatment cycle, T4 = after 1 mo follow-up, VAS = Visual Analog Scale.

Table 3.

Percentage changes with respect to baseline for Visual Analog Scale (VAS), Fibromyalgia Impact Questionnaire (FIQ), and Fibromyalgia Assessment Scale (FAS) scores ( $p$-values are in bold if statistically significant after Bonferroni correction).

\begin{tabular}{|c|c|c|c|c|c|}
\hline Scale & $\begin{array}{l}\text { Group and } \\
\text { Comparison }\end{array}$ & T1 vs T0 & T2 vs T0 & T3 vs T0 & T4 vs T0 \\
\hline & Sham-ELF & $8.0 \pm 25.5$ & $6.3 \pm 16.0$ & $-57.0 \pm 25.8$ & $-39.7 \pm 26.0$ \\
\hline \multirow[t]{3}{*}{ FAS } & ELF-Sham & $-39.7 \pm 16.2$ & $-46.5 \pm 17.3$ & $-11.8 \pm 18.9$ & $-1.2 \pm 15.4$ \\
\hline & Sham-ELF & $-0.7 \pm 20.9$ & $-4.5 \pm 20.8$ & $-39.3 \pm 18.4$ & $-46.9 \pm 22.8$ \\
\hline & $p$-Value & $<0.001$ & $<0.001$ & 0.09 & $<0.001$ \\
\hline & $p$-Value & $<0.001$ & $<0.001$ & 0.001 & $<0.001$ \\
\hline
\end{tabular}

$\overline{E L F}=$ extremely low-frequency (magnetic field), T0 = baseline, $\mathrm{T} 1=$ end of first treatment cycle, $\mathrm{T} 2$ = beginning of second treatment cycle (after 1 mo washout), $\mathrm{T} 3=$ end of second treatment cycle, $\mathrm{T} 4=$ after 1 mo follow-up.

on peripheral neural stimulation and regulates microcirculation, like laser therapy [48], interrupting pain mechanisms and promoting analgesia.

Based on our results, future studies in this field should increase the sample size and extend the observation times (up to $1 \mathrm{yr}$ ) and also include other overlapping painful conditions with FM.

\section{CONCLUSIONS}

ELF-MF therapy, within the parameters of this treatment protocol, can be recommended as part of a multimodal approach to reducing pain in FM subjects for short periods and to intensifying the results of drug therapy or physiother- apy. ELF-MFs have analgesic effects in FM. Clinically, determining the biological effects of ELF-MF exposure in FM could facilitate the development of alternative treatments and novel therapeutic tools. However, future research is needed to determine the long-term repeatability of various treatment protocols, which requires greater standardization with regard to patient safety and the duration of the effects.

\section{ACKNOWLEDGMENTS}

\section{Author Contributions:}

Study concept and design: T. Paolucci, G. Piccinini. Acquisition of data: C. Piermattei, S. de Angelis, M. R. Grasso. Analysis and interpretation of data: M. Iosa, F. Zangrando, C. Piermattei. 
Drafting of manuscript: T. Paolucci, G. Piccinini.

Critical revision of manuscript for important intellectual content:

V. M. Saraceni, T. Paolucci, G. Piccinini.

Statistical analysis: M. Iosa.

Study supervision: V. M. Saraceni, F. Zangrando.

Financial Disclosures: The authors have declared that no competing interests exist.

Funding/Support: This material was unfunded at the time of manuscript preparation.

Institutional Review: This study was approved by the ethical committee of Sapienza University of Rome (registration number 3295, protocol number 844/14, ClinicalTrials.gov identifier NCT02231541). All subjects gave written informed consent after receiving detailed information about the study's aims and procedures per the Declaration of Helsinki.

Participant Follow-Up: The authors have no plans to notify the study subjects of the publication of this article because of a lack of contact information.

\section{REFERENCES}

1. Wolfe F, Clauw DJ, Fitzcharles MA, Goldenberg DL, Katz RS, Mease P, Russell AS, Russell IJ, Winfield JB, Yunus MB. The American College of Rheumatology preliminary diagnostic criteria for fibromyalgia and measurement of symptom severity. Arthritis Care Res (Hoboken). 2010; 62(5):600-10. [PMID:20461783]

http://dx.doi.org/10.1002/acr.20140

2. Wolfe F, Ross K, Anderson J, Russell IJ, Hebert L. The prevalence and characteristics of fibromyalgia in the general population. Arthritis Rheum. 1995;38(1):19-28.

[PMID:7818567] http://dx.doi.org/10.1002/art.1780380104

3. Malin K, Littlejohn GO. Psychological control is a key modulator of fibromyalgia symptoms and comorbidities. J Pain Res. 2012;5:463-71. [PMID:23152697]

4. Jones KD, Deodhar P, Lorentzen A, Bennett RM, Deodhar AA. Growth hormone perturbations in fibromyalgia: A review. Semin Arthritis Rheum. 2007;36(6):357-79.

[PMID:17224178]

http://dx.doi.org/10.1016/j.semarthrit.2006.09.006

5. Di Franco M, Iannuccelli C, Valesini G. Neuroendocrine immunology of fibromyalgia. Ann N Y Acad Sci. 2010; 1193:84-90. [PMID:20398012] http://dx.doi.org/10.1111/j.1749-6632.2009.05344.x

6. Craig AD. Pain mechanisms: Labeled lines versus convergence in central processing. Annu Rev Neurosci. 2003;26: 1-30. [PMID:12651967]

http://dx.doi.org/10.1146/annurev.neuro.26.041002.131022

7. Arnold LM, Williams DA, Hudson JI, Martin SA, Clauw DJ, Crofford LJ, Wang F, Emir B, Lai C, Zablocki R, Mease PJ. Development of responder definitions for fibromyalgia clinical trials. Arthritis Rheum. 2012;64(3):885-
94. [PMID:21953205]

http://dx.doi.org/10.1002/art.33360

8. Johannsson V. Does a fibromyalgia personality exist? J Musculoskelet Pain. 1993;1:245-52.

http://dx.doi.org/10.1300/J094v01n03 26

9. Cedraschi C, Girard E, Luthy C, Kossovsky M, Desmeules J, Allaz AF. Primary attributions in women suffering fibromyalgia emphasize the perception of a disruptive onset for a long-lasting pain problem. J Psychosom Res. 2013; 74(3):265-69. [PMID:23438720]

http://dx.doi.org/10.1016/j.jpsychores.2012.12.014

10. Cherry BJ, Zettel-Watson L, Chang JC, Shimizu R, Rutledge DN, Jones CJ. Positive associations between physical and cognitive performance measures in fibromyalgia. Arch Phys Med Rehabil. 2012;93(1):62-71. [PMID:21996532] http://dx.doi.org/10.1016/j.apmr.2011.08.006

11. Kaleth AS, Saha CK, Jensen MP, Slaven JE, Ang DC. Effect of moderate to vigorous physical activity on longterm clinical outcomes and pain severity in fibromyalgia. Arthritis Care Res (Hoboken). 2013;65(8):1211-18. [PMID:23401486] http://dx.doi.org/10.1002/acr.21980

12. Köllner V, Häuser W, Klimczyk K, Kühn-Becker H, Settan M, Weigl M, Bernardy K; Arbeitsgemeinschaft der Wissenschaftlichen Medizinischen Fachgesellschaften. [Psychotherapy for patients with fibromyalgia syndrome. Systematic review, meta-analysis and guideline]. Schmerz. 2012;26(3):291-96. German. [PMID:22760462]

13. Carville SF, Arendt-Nielsen L, Bliddal H, Blotman F, Branco JC, Buskila D, Da Silva JA, Danneskiold-Samsøe B, Dincer F, Henriksson C, Henriksson KG, Kosek E, Longley K, McCarthy GM, Perrot S, Puszczewicz M, Sarzi-Puttini P, Silman A, Späth M, Choy EH; EULAR. EULAR evidence-based recommendations for the management of fibromyalgia syndrome. Ann Rheum Dis. 2008; 67(4):536-41. [PMID:17644548] http://dx.doi.org/10.1136/ard.2007.071522

14. Bidonde J, Busch AJ, Bath B, Milosavljevic S. Exercise for adults with fibromyalgia: An umbrella systematic review with synthesis of best evidence. Curr Rheumatol Rev. 2014;10(1):45-79. [PMID:25229499] http://dx.doi.org/10.2174/1573403X10666140914155304

15. Winkelmann A, Häuser W, Friedel E, Moog-Egan M, Seeger D, Settan M, Weiss T, Schiltenwolf M; Arbeitsgemeinschaft der Wissenschaftlichen Medizinischen Fachgesellschaften. [Physiotherapy and physical therapies for fibromyalgia syndrome. Systematic review, meta-analysis and guideline]. Schmerz. 2012;26(3):276-86. German. [PMID:22760460] http://dx.doi.org/10.1007/s00482-012-1171-3

16. Ruaro JA, Fréz AR, Ruaro MB, Nicolau RA. Low-level laser therapy to treat fibromyalgia. Lasers Med Sci. 2014; 
29(6):1815-19. [PMID:24801056]

http://dx.doi.org/10.1007/s10103-014-1566-8

17. Quittan M, Schuhfried O, Wiesinger GF, Fialka-Moser V. [Clinical effectiveness of magnetic field therapy - a review of the literature]. Acta Med Austriaca. 2000; 27(3):61-68. German. [PMID:10897384]

18. Shafiei SA, Firoozabadi SM, Tabatabaie KR, Ghabaee M. Investigation of EEG changes during exposure to extremely low-frequency magnetic field to conduct brain signals. Neurol Sci. 2014;35(11):1715-21. [PMID:24864004] http://dx.doi.org/10.1007/s10072-014-1819-0

19. Monazzam MR, Hosseini M, Matin LF, Aghaei HA, Khosroabadi H, Hesami A. Sleep quality and general health status of employees exposed to extremely low frequency magnetic fields in a petrochemical complex. J Environ Health Sci Eng. 2014 Apr 29;12:78.

20. Bistolfi F. Extremely low-frequency pulsed magnetic fields and multiple sclerosis: Effects on neurotransmission alone or also on immunomodulation? Building a working hypothesis. Neuroradiol J. 2007;20(6):676-93. [PMID:24300003] http://dx.doi.org/10.1177/197140090702000612

21. Mild KH, Mattsson MO. ELF noise fields: A review. Electromagn Biol Med. 2010;29(3):72-97. [PMID:20707642] http://dx.doi.org/10.3109/15368378.2010.482487

22. Reale M, Kamal MA, Patruno A, Costantini E, D'Angelo C, Pesce M, Greig NH. Neuronal cellular responses to extremely low frequency electromagnetic field exposure: implications regarding oxidative stress and neurodegeneration. PLoS One. 2014 Aug 15;9(8):e104973. [PMID:25127118]

23. Rauš Balind S, Selaković V, Radenović L, Prolić Z, Janać B. Extremely low frequency magnetic field $(50 \mathrm{~Hz}, 0.5$ $\mathrm{mT}$ ) reduces oxidative stress in the brain of gerbils submitted to global cerebral ischemia. PLoS One. 2014;9(2): e88921. [PMID:24586442]

24. Shafiei SA, Firoozabadi SM. Local ELF-magnetic field: A possible novel therapeutic approach to psychology symptoms. Neurol Sci. 2014;35(11):1651-56.

[PMID:25073696] http://dx.doi.org/10.1007/s10072-014-1905-3

25. Weintraub MI, Cole SP. Pulsed magnetic field therapy in refractory neuropathic pain secondary to peripheral neuropathy: Electrodiagnostic parameters-pilot study. Neurorehabil Neural Repair. 2004;18(1):42-46.

[PMID:15035963] http://dx.doi.org/10.1177/0888439003261024

26. Musaev AV, Guseinova SG, Imamverdieva SS. The use of pulsed electromagnetic fields with complex modulation in the treatment of patients with diabetic polyneuropathy. Neurosci Behav Physiol. 2003;33(8):745-52.

[PMID:14635988] http://dx.doi.org/10.1023/A:1025184912494
27. Sandyk R. Application of weak electromagnetic fields facilitates sensory-motor integration in patients with multiple sclerosis. Int J Neurosci. 1996;85(1-2):101-10. [PMID:8727686] http://dx.doi.org/10.3109/00207459608986355

28. Shupak NM, McKay JC, Nielson WR, Rollman GB, Prato FS, Thomas AW. Exposure to a specific pulsed low-frequency magnetic field: A double-blind placebo-controlled study of effects on pain ratings in rheumatoid arthritis and fibromyalgia patients. Pain Res Manag. 2006;11(2):85-90. [PMID:16770449] http://dx.doi.org/10.1155/2006/842162

29. Maestú C, Blanco M, Nevado A, Romero J, RodríguezRubio P, Galindo J, Bautista Lorite J, de las Morenas F, Fernández-Argüelles P. Reduction of pain thresholds in fibromyalgia after very low-intensity magnetic stimulation: A double-blinded, randomized placebo-controlled clinical trial. Pain Res Manag. 2013;18(6):e101-06. [PMID:24308025] http://dx.doi.org/10.1155/2013/270183

30. Thomas AW, Graham K, Prato FS, McKay J, Forster PM, Moulin DE, Chari S. A randomized, double-blind, placebocontrolled clinical trial using a low-frequency magnetic field in the treatment of musculoskeletal chronic pain. Pain Res Manag. 2007;12(4):249-58. [PMID:18080043] http://dx.doi.org/10.1155/2007/626072

31. Sutbeyaz ST, Sezer N, Koseoglu F, Kibar S. Low-frequency pulsed electromagnetic field therapy in fibromyalgia: A randomized, double-blind, sham-controlled clinical study. Clin J Pain. 2009;25(8):722-28. [PMID:19920724] http://dx.doi.org/10.1097/AJP.0b013e3181a68a6c

32. Bennett R. The Fibromyalgia Impact Questionnaire (FIQ): A review of its development, current version, operating characteristics and uses. Clin Exp Rheumatol. 2005;23(5 Suppl 39):S154-62. [PMID:16273800]

33. Sarzi-Puttini P, Atzeni F, Fiorini T, Panni B, Randisi G, Turiel M, Carrabba M. Validation of an Italian version of the Fibromyalgia Impact Questionnaire (FIQ-I). Clin Exp Rheumatol. 2003;21(4):459-64. [PMID:12942697]

34. Salaffi F, Sarzi-Puttini P, Girolimetti R, Gasparini S, Atzeni F, Grassi W. Development and validation of the self-administered Fibromyalgia Assessment Status: A disease-specific composite measure for evaluating treatment effect. Arthritis Res Ther. 2009;11(4):R125.

[PMID:19686606] http://dx.doi.org/10.1186/ar2792

35. Fries JF, Spitz P, Kraines RG, Holman HR. Measurement of patient outcome in arthritis. Arthritis Rheum. 1980;23(2): 137-45. [PMID:7362664] http://dx.doi.org/10.1002/art.1780230202

36. Huskisson EC. Measurement of pain. Lancet. 1974; 2(7889):1127-31. [PMID:4139420] http://dx.doi.org/10.1016/S0140-6736(74)90884-8 
37. Todd KH, Funk JP. The minimum clinically important difference in physician-assigned visual analog pain scores. Acad Emerg Med. 1996;3(2):142-46. [PMID:8808375] http://dx.doi.org/10.1111/j.1553-2712.1996.tb03402.x

38. Lee JS, Hobden E, Stiell IG, Wells GA. Clinically important change in the visual analog scale after adequate pain control. Acad Emerg Med. 2003;10(10):1128-30.

[PMID:14525749]

http://dx.doi.org/10.1111/j.1553-2712.2003.tb00586.x

39. Prato FS, Carson JJ, Ossenkopp KP, Kavaliers M. Possible mechanisms by which extremely low frequency magnetic fields affect opioid function. FASEB J. 1995;9(9):807-14.

40. Del Seppia C, Ghione S, Luschi P, Ossenkopp KP, Choleris E, Kavaliers M. Pain perception and electromagnetic fields. Neurosci Biobehav Rev. 2007;31(4):619-42.

41. Bradley LA. Pathophysiology of fibromyalgia. Am J Med. 2009;122(12, Suppl):S22-30. [PMID:19962493] http://dx.doi.org/10.1016/j.amjmed.2009.09.008

42. Burnstock G, Arnett TR, Orriss IR. Purinergic signalling in the musculoskeletal system. Purinergic Signal. 2013; 9(4):541-72. [PMID:23943493] http://dx.doi.org/10.1007/s11302-013-9381-4

43. Bazzichi L, Giannaccini G, Betti L, Fabbrini L, Schmid L, Palego L, Giacomelli C, Rossi A, Giusti L, De Feo F, Giuliano T, Mascia G, Bombardieri S, Lucacchini A. ATP, calcium and magnesium levels in platelets of patients with primary fibromyalgia. Clin Biochem. 2008;41(13):1084-90. [PMID:18634773] http://dx.doi.org/10.1016/j.clinbiochem.2008.06.012

44. Engström S, Fitzsimmons R. Five hypotheses to examine the nature of magnetic field transduction in biological systems. Bioelectromagnetics. 1999;20(7):423-30.

[PMID:10495307]

http://dx.doi.org/10.1002/(SICI)1521186X(199910)20:7<423::AID-BEM3>3.0.CO;2-W

45. Ongaro A, Varani K, Masieri FF, Pellati A, Massari L, Cadossi R, Vincenzi F, Borea PA, Fini M, Caruso A, De Mattei M. Electromagnetic fields (EMFs) and adenosine receptors modulate prostaglandin $\mathrm{E}(2)$ and cytokine release in human osteoarthritic synovial fibroblasts. J Cell Physiol.
2012;227(6):2461-69. [PMID:21830213]

http://dx.doi.org/10.1002/jep.22981

46. Aaron RK, Boyan BD, Ciombor DM, Schwartz Z, Simon BJ. Stimulation of growth factor synthesis by electric and electromagnetic fields. Clin Orthop Relat Res. 2004;(419): 30-37. [PMID:15021128] http://dx.doi.org/10.1097/00003086-200402000-00006

47. Kavaliers M, Ossenkopp KP, Tysdale DM. Evidence for the involvement of protein kinase $\mathrm{C}$ in the modulation of morphine-induced 'analgesia' and the inhibitory effects of exposure to $60-\mathrm{Hz}$ magnetic fields in the snail, Cepaea nemoralis. Brain Res. 1991;554(1-2):65-71.

[PMID:1933319] http://dx.doi.org/10.1016/0006-8993(91)90172-R

48. de Carvalho PT, Leal-Junior EC, Alves AC, Rambo CS, Sampaio LM, Oliveira CS, Albertini R, de Oliveira LV. Effect of low-level laser therapy on pain, quality of life and sleep in patients with fibromyalgia: Study protocol for a double-blinded randomized controlled trial. Trials. 2012; 13:221. [PMID:23171567]

http://dx.doi.org/10.1186/1745-6215-13-221

Submitted for publication April 8, 2015. Accepted in revised from November 3, 2015.

This article and any supplementary material should be cited as follows:

Paolucci T, Piccinini G, Iosa M, Piermattei C, de Angelis S, Grasso MR, Zangrando F, Saraceni VM. Efficacy of extremely low-frequency magnetic field in fibromyalgia pain: A pilot study. J Rehabil Res Dev. 2016;53(6):102334.

http://dx.doi.org/10.1682/JRRD.2015.04.0061

ORCID: Teresa Paolucci, MD, PhD: 0000-0002-86941404

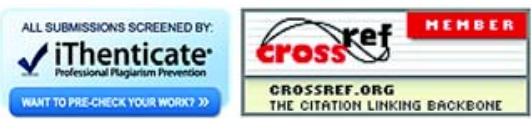

